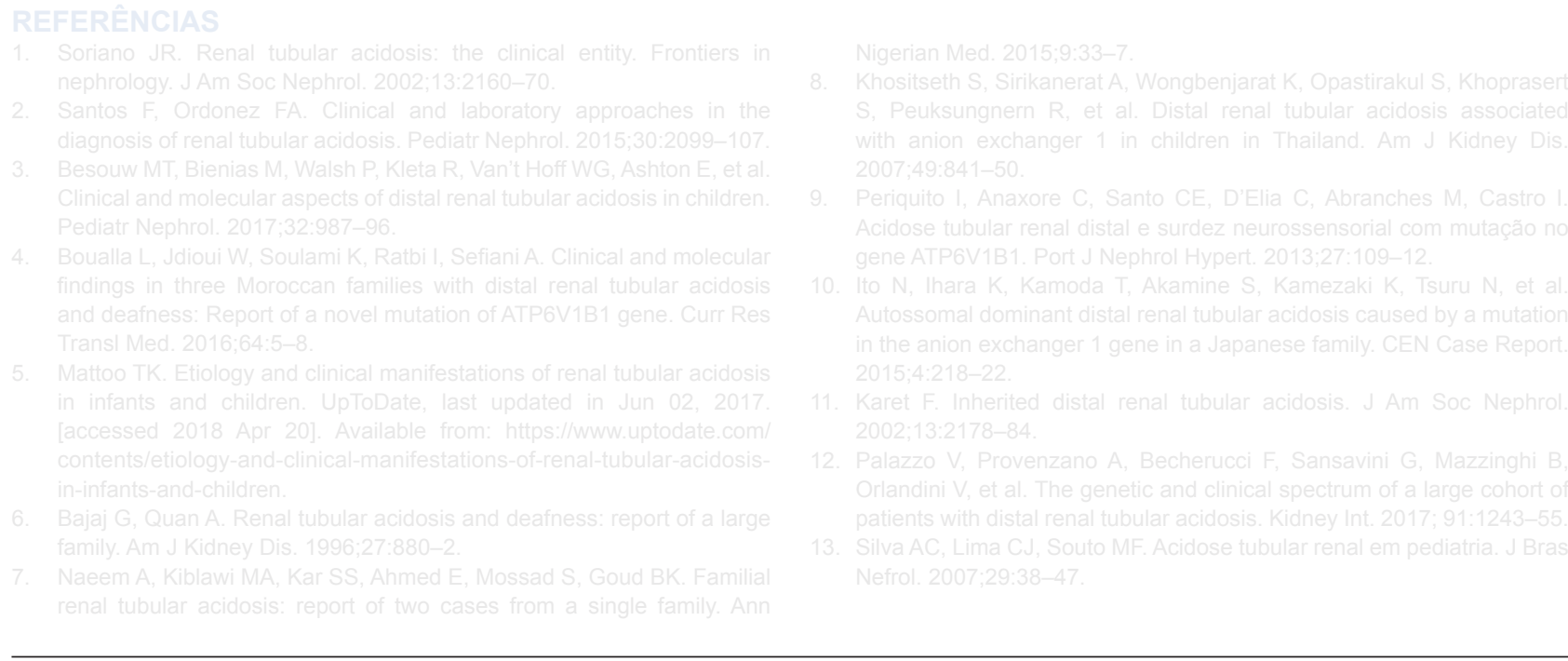

\title{
Taquicardia Sinusal em Adolescentes: A Importância do Diagnóstico Diferencial
}

\section{Sinus Tachycardia in the Adolescent: The Importance of Differential Diagnosis}

\author{
Inês OLIVEIRA $\rrbracket^{1}$, Hugo TELES ${ }^{1}$, Filipe SEIXO ${ }^{2}$, Anabela FERRÃO $^{3}$ \\ Acta Med Port 2019 Jul-Aug;32(7-8):545-548 - https://doi.org/10.20344/amp.10719
}

\section{RESUMO}

A taquicardia sinusal é uma manifestação comum em Pediatria, associada frequentemente a condições fisiológicas, nomeadamente as síndromes de somatização. Pode ser um sinal precoce de patologias graves, com prognóstico adverso. Adolescente de 15 anos, sexo feminino, saudável e sob anticoncetivos orais desde há um mês. Admitida no serviço de urgência por lipotimia. Estava assintomática e não apresentava alterações ao exame objetivo, à exceção de taquicardia sinusal persistente, apesar da estabilidade hemodinâmica. A angio-tomografia computorizada torácica revelou trombos nas artérias pulmonares principais, confirmando o diagnóstico de embolia pulmonar bilateral. Foi iniciada hipocoagulação com boa evolução. A embolia pulmonar em idade pediátrica é rara, embora provavelmente sub-diagnosticada pela apresentação clínica inespecífica, que pode conduzir ao atraso diagnóstico e, consequentemente, agravar o prognóstico.

Palavras-chave: Adolescente; Anticoncepcionais; Diagnóstico Diferencial; Embolia Pulmonar; Taquicardia Sinusal

\section{ABSTRACT}

Sinus tachycardia is common in pediatric age, and is usually related to benign physiological conditions, such as somatoform disorders. Nevertheless, it can also be a presenting sign of a disease with an ominous prognosis. We present a case of a previously healthy 15-year-old girl, admitted for syncope. She had been well until one week before admission, when thoracic pain developed. One month prior to the admission she had started oral contraceptives. On examination, she had persistent sinus tachycardia, despite hemodynamic stability and no other associated sign or symptom. Thoracic computed tomography angiography revealed thrombi in the main pulmonary arteries, which confirmed bilateral pulmonary embolism. She was started on anticoagulation with unremarkable clinical course evolution. Pediatric pulmonary embolism is rare, although probably underdiagnosed, given the nonspecific presentation in most cases. Maintaining a high level of clinical suspicion of pulmonary embolism avoids delay in the diagnosis, allows early appropriate treatment and improves prognosis.

Keywords: Adolescent; Contraceptive Agents; Diagnosis, Differential; Pulmonary Embolism; Tachycardia, Sinus/diagnosis

\section{INTRODUÇÃO}

A embolia pulmonar (EP) em Pediatria é rara. Os registos diferem quanto às incidências estimadas, provavelmente resultado da dificuldade diagnóstica desta entidade em idade pediátrica. A incidência estimada de tromboembolismo venoso (TEV) é de cerca de 58 casos por cada 10000 admissões hospitalares, correspondendo a EP a $11 \%$ destes. ${ }^{1} \mathrm{~A}$ distribuição é bimodal, com maior incidência no primeiro ano de vida e nos adolescentes, não parecendo existir um predomínio de género. ${ }^{2}$

1. Serviço de Pediatria. Hospital de São Bernardo. Centro Hospitalar de Setúbal. Setúbal. Portugal.

2. Serviço de Cardiologia. Hospital de São Bernardo. Centro Hospitalar de Setúbal. Setúbal. Portugal.

3. Unidade de Hematologia Pediátrica. Departamento de Pediatria. Hospital de Santa Maria. Centro Hospitalar Lisboa Norte. Lisboa. Portugal.

$\triangle$ Autor correspondente: Inês Oliveira. inesfigueiredoliveira@gmail.com

Recebido: 24 de abril de 2018 - Aceite: 03 de setembro de 2018 | Copyright $\odot$ Ordem dos Médicos 2019 
É, no entanto, provavelmente sub-diagnosticada, pela apresentação clínica inespecífica e silenciosa na maior parte dos casos. ${ }^{2-4}$ A mortalidade por TEV é elevada, podendo atingir os $23 \%$, particularmente quando relacionada com cateter. ${ }^{5}$ A deteção é tendencialmente tardia, com tempos médios de diagnóstico de sete dias após a admissão hospitalar. ${ }^{6}$

Os scores de Wells ${ }^{7}$ e pulmonary embolism rule-out criteria (PERC), ${ }^{8}$ utilizados em adultos para determinação do risco de EP não estão validados para utilização em Pediatria. Um estudo recente avaliou a sua aplicação a uma população pediátrica, tendo o primeiro demonstrado uma sensibilidade de $86 \%$ e especificidade de $60 \%$ e o segundo de $100 \%$ e $24 \%$, respetivamente. Trata-se porém, de um estudo realizado num único centro, com uma amostra de pequeno volume. ${ }^{9}$

Aproximadamente $90 \%$ das tromboses venosas em idade pediátrica ocorrem na presença de fatores predisponentes. O mais importante é a presença de cateter venoso central (CVC), isoladamente ou em associação a outros fatores como neoplasia e quimioterapia, cardiopatia congénita, história familiar de TEV e trombofilias hereditárias, ${ }^{3}$ obesidade, imobilidade prolongada, nutrição parentérica, lúpus eritematoso sistémico (LES), síndrome nefrótico, cirurgia ou trauma..$^{10} \mathrm{Em}$ adolescentes do género feminino, a combinação de hábitos tabágicos e início recente de anticoncetivos orais (ACO) com base em estrogénios deve reduzir o limiar de suspeita diagnóstica, sendo o reconhecimento precoce crucial no prognóstico destes casos.

\section{CASO CLÍNICO}

Adolescente de sexo feminino, 15 anos, previamente saudável, não fumadora, que iniciou ACO um mês antes. Admitida no serviço de urgência por lipotimia em repouso, presenciada, sem pródromos, sem perda de conhecimento, de curta duração (inferior a cinco minutos) e recuperação espontânea. Referia toracalgia, episódica com uma semana de evolução, de características pleuríticas, localização retroesternal, sem irradiação, intensidade 5/10, agravada com esforços, sem fatores de alívio, sem sintomas acompanhantes. Negava história de traumatismo ou imobilização prolongada recente e antecedentes familiares relevantes.

À admissão estava ansiosa, apirética e sem dor. Eupneica, SpO2 100\% em ar ambiente, taquicárdica [130 bpm, para um limite superior do normal (LSN) de 100 bpm], pressão arterial média $72 \mathrm{mmHg}$, com sistólica e diastólica de 101/57 mmHg, respetivamente. Não apresentava sinais de desidratação e tinha adequada perfusão periférica. Pulsos periféricos amplos, taquicardicos, simétricos, regulares. Auscultação cardiopulmonar sem alterações. Abdómen mole, depressível, indolor e membros inferiores sem edema ou sinais de trombose venosa superficial ou profunda. Foi realizada terapêutica ansiolítica (diazepam per os). Durante a vigilância manteve taquicardia sinusal, sem fator perpetuador aparente. Analiticamente, apresentava anemia microcítica hipocrómica ( $\mathrm{Hb} 11 \mathrm{~g} / \mathrm{dL}$ ), sem elevação dos parâmetros inflamatórios, função tiroideia normal, gasimetria arterial sem insuficiência respiratória e com normocapnia. O doseamento de tóxicos na urina foi negativo e a telerradiografia do tórax sem alterações. Eletrocardiograma de 12 derivações (ECG) com taquicardia sinusal ( 128bpm), eixo normal, padrão S1Q3T3, sem outras alterações da repolarização ventricular (Fig. 1).

Perante taquicardia inapropriada persistente, colocou-se a hipótese de tromboembolia pulmonar (TEP). Os D-dímeros estavam aumentados $(2757 \mathrm{ng} / \mathrm{mL}$, para um LSN de $230 \mathrm{ng} / \mathrm{mL}$ ) e a angio-tomografia computorizada (TC) torácica identificou trombos nas artérias pulmonares principais bilateralmente (Fig. 2).

Foi realizado ecocardiograma transtorácico, que evidenciou dilatação das câmaras cardíacas direitas e sinais de sobrecarga de pressão do ventrículo direito, com retilinização sisto-diastólica do septo interventricular, insuficiência

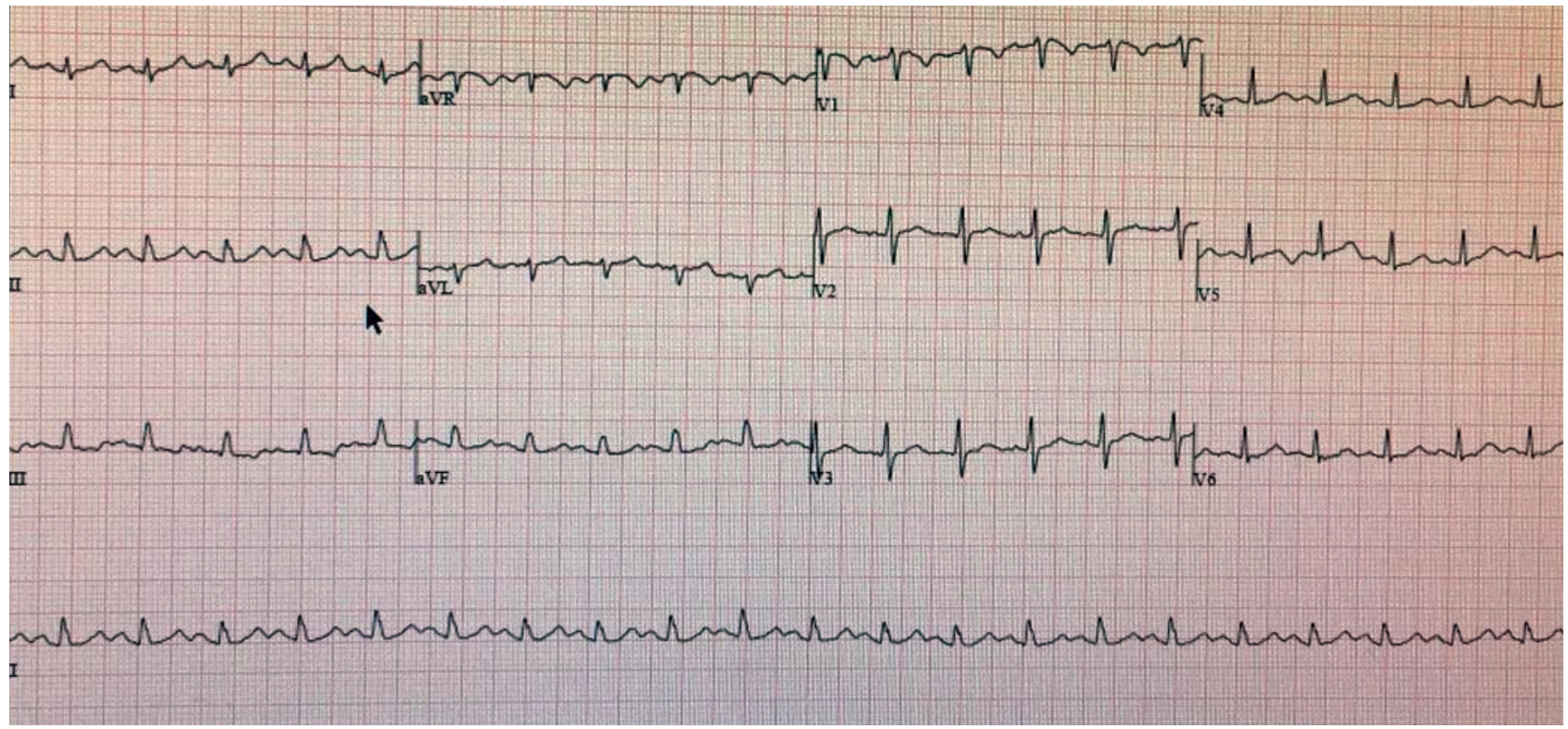

Figura 1 - Eletrocardiograma de 12 derivações. Taquicardia sinusal e sinal de McGinn-White, padrão com onda S em DI, onda Q e onda T invertida em DIII (S1Q3T3). 


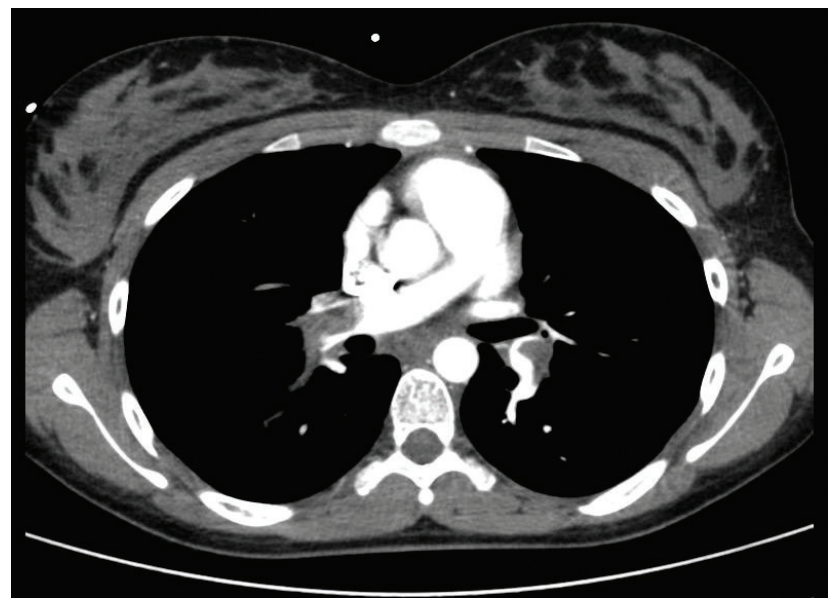

Figura 2 - Angio-TC pulmonar de alta resolução. Evidentes formações endoluminais volumosas nos ramos principais direito e esquerdo da artéria pulmonar, compatível com tromboembolismo pulmonar bilateral.

tricúspide moderada, com pressão sistólica da artéria pulmonar estimada em $37 \mathrm{mmHg}$. Foram avaliados marcadores de necrose miocárdica (elevados, troponina I 0,25 ng/ $\mathrm{mL}$, para um LSN de $0,04 \mathrm{ng} / \mathrm{mL}$ ).

Após o diagnóstico, apurou-se história de TEV dos membros inferiores e pulmonar em tia materna jovem com mutação $A 1298 \mathrm{C}$ em heterozigotia do gene metilenotetrahidrofolato redutase (MTHFR) e polimorfismo $4 \mathrm{G} / 4 \mathrm{G}$ do inibidor do ativador do plasminogénio tipo 1 (PAl-1).

Foi iniciada hipocoagulação com heparina de baixo peso molecular (enoxaparina $1 \mathrm{mg} / \mathrm{kg}$ de 12/12horas) e efetuada transferência para a unidade de cuidados intensivos pediátricos atendendo a TEP de risco intermédio-alto, com sinais de sobrecarga ventricular direita e elevação de marcadores de lesão miocárdica, com risco potencial de descompensação hemodinâmica. Manteve-se sem insuficiência respiratória ou outro sinal de disfunção de órgão, hemodinamicamente estável e com resolução da hipertensão pulmonar em ecocardiograma transtorácico de reavaliação ao $10^{\circ}$ dia de internamento, altura em que teve alta, hipocoagulada com antagonista da vitamina K (varfarina) ajustado a INR terapêutico $(2,0-3,0)$. Do estudo complementar, foi identificada ligeira hiperhomocisteinemia (21 mmol/L, LSN 13,9 mmol/L), diminuição ligeira não significativa dos níveis plasmáticos da proteína S (45\%), com restante estudo de trombofilias negativo.

Até ao $12^{\circ}$ mês de acompanhamento clínico, manteve-se assintomática, sem restrição das atividades de vida diária ou limitação para esforços de média/elevada intensidade e sem recorrência de eventos tromboembólicos venosos ou arteriais sob hipocoagulação oral. O ACO baseado em estrogénios manteve-se suspenso, tendo sido orientada para consulta de planeamento familiar para decisão da estratégia futura.

\section{DISCUSSÃO}

A taquicardia sinusal é uma manifestação frequente em Pediatria, associada na maioria das vezes a condições fisiológicas, benignas. As principais causas são febre, anemia, desidratação ou perturbações da ansiedade. Embora inespecífico, pode ser um sinal precoce de patologias com prognóstico desfavorável, pelo que a sua identificação não deve ser desvalorizada, particularmente quando persistente e inapropriada, isto é, em repouso, sem fator precipitante claro e na ausência de cardiopatia estrutural aparente. O diagnóstico diferencial é vasto, exigindo elevado índice de suspeita clínica, nomeadamente para causas menos frequentes em idade pediátrica, como a EP, cuja prevalência parece estar a aumentar. Apesar da tendência de crescimento, maioritariamente em relação com o aumento da sobreviva de crianças com doenças crónicas, ${ }^{9}$ mantém-se rara, particularmente em crianças saudáveis.

$\mathrm{O}$ início recente de ACO (< três meses) está descrito como um dos mais significativos fatores de risco para EP neste grupo e a taquicardia sinusal como o sinal mais frequente. ${ }^{11}$ Foi esta associação, particularmente a taquicardia inapropriada persistente, que constituiu o principal sinal de alarme e levantou a suspeita de EP, numa altura em que era ainda desconhecida a história familiar.

Inicialmente foi considerada a hipótese de perturbação da ansiedade, dada a pouca especificidade das queixas e a sua associação a síndromes de somatização, tão comuns no adolescente, o que pode protelar o diagnóstico.

$\mathrm{Na}$ investigação diagnóstica, os d-dímeros, produto da degradação da fibrina, poderão ser úteis perante a suspeita clínica de EP. No entanto, apesar da sensibilidade para excluir EP no adulto, particularmente de risco não alto, o seu poder discriminatório em idade pediátrica é limitado, sendo reportados valores normais em cerca de $15 \%-40 \%$ das crianças com EP. ${ }^{12}$ Atualmente, a angioTC pulmonar de alta resolução constitui o método de diagnóstico de escoIha pela sua elevada sensibilidade e especificidade, apesar das limitações inerentes em idade pediátrica, nomeadamente a exposição à radiação ionizante e eventual necessidade de sedação. ${ }^{13}$

A estratificação de risco foi realizada através do electrocardiograma e ecocardiograma, que revelaram sinais de sobrecarga cardíaca direita, e dos marcadores de necrose miocárdica, com elevação da troponina, o que classificaria a EP aguda como de risco intermédio-alto para mortalidade precoce, de acordo com os sistemas de estratificação de risco utilizados no adulto. ${ }^{7,8}$ Esta classificação, porém, não é realizada frequentemente na criança, sobretudo porque escasseiam estudos que avaliem o valor prognóstico destes achados nesta população. ${ }^{12} \mathrm{Um}$ estudo demonstrou que a disfunção cardíaca aguda com evidência de sobrecarga direita é comum na EP pediátrica, porém, a morte e disfunção crónica não é frequente quando instituída terapêutica antitrombótica atempada e agressiva. ${ }^{14}$

Apesar dos sinais de sobrecarga cardíaca direita, a adolescente manteve-se hemodinamicamente estável, razão pela qual se optou por instituir hipocoagulação oral e não realizar fibrinólise.

Atualmente, as recomendações internacionais recomendam a investigação complementar de trombofilias em 
todas as crianças com história de EP, embora esteja ainda por esclarecer a sua relevância na estratificação do risco, terapêutica e prognóstico.

A evolução neste caso foi favorável, o que está de acordo com a literatura, com risco de recorrência de EP sintomática descrito em cerca de $9 \%$ dos casos e persistência de defeitos de perfusão aos seis meses pós-evento em $18 \% .{ }^{14}$ Estes outcomes adversos associaram-se à presença de condições inflamatórias crónicas não infeciosas como o LES ou a colite ulcerosa. ${ }^{14}$

O TEV na adolescência, particularmente no género feminino, tem implicações importantes no que diz respeito à estratégia contracetiva. Estudos prospetivos mostraram risco acrescido de TEV recorrente com ACO combinados contendo estrogénios (risco absoluto $13,9 \%$ por ano), pelo que a sua utilização após evento tromboembólico está contra-indicada. Estratégias alternativas devem ser discutidas com a adolescente, sendo opções a considerar os métodos barreira, dispositivos intra-uterinos (DIU) sem fármaco, embora os DIU com levonorgestrel pareçam também ser seguros, ou anticoncetivos baseados em progestagénios (orais ou subcutâneo). ${ }^{15}$

Pretendemos alertar para uma entidade rara no adolescente, mas com desfecho potencialmente grave. A inexistência de scores de estratificação de risco específicos para a idade pediátrica permanece uma carência, sendo necessários estudos multicêntricos que sirvam de base

\section{REFERÊNCIAS}

1. Raffini L, Huang YS, Witmer C, Feudtner C. Dramatic increase in venous thromboembolism in children's hospital in the United States from 2001 to 2007. Pediatrics. 2009;124:1001-8.

2. Stein PD, Kayali F, Olson RE. Incidence of venous thromboembolism in infants and children: data from the National Hospital Discharge Survey. J Pediatr. 2004;145:563-5.

3. Tavil B, Kuskonmaz B, Kiper N, Cetin M, Gumruk F, Gurgey A. Pulmonary thromboembolism in childhood: A single-center experience from Turkey. Heart Lung. 2009;38:56-65.

4. Brandão LR, Labarque V, Diab Y, Williams S, Manson DE. Pulmonary embolism in children. Semin Thromb Hemost. 2011;37:772-85.

5. Monagle $\mathrm{P}$, Adams $\mathrm{M}$, Mahoney $\mathrm{M}$, Bernstein $\mathrm{ML}$, Halton JM Massicote $\mathrm{P}$, et al. Outcome of pediatric thromboembolic disease: a report from the Canadian Childhood Thrombophilia Registry. Pediatr Res. 2000;47:763-6.

6. Rajpurkar M, Warrier I, Chitlur M, Sabo C, Frey MJ, Hollon W, et al. Pulmonary embolism-experience at a single children's hospital. Thromb Res. 2007;119:699-703.

7. Wolf SJ, McCubbin TR, Feldhaus KM, Faragher JP, Adcock DM. Prospective validation of Wells criteria in evaluation of patients with suspected pulmonary embolism. Ann Emerg Med. 2004;44:503-10.

8. Wolf SJ, McCubbin TR, Nordenholz KE, Naviaux NW, Haukoos JS. Assessment of the Pulmonary Embolism Rule-out Criteria rule for ao desenvolvimento de um sistema de decisão clínica que auxilie na determinação fiável e reprodutível da probabilidade pré-teste de EP, sensibilizando os profissionais de saúde na sua investigação diagnóstica e intervenção terapêutica.

\section{PROTECÇÃO DE PESSOAS E ANIMAIS}

Os autores declaram que os procedimentos seguidos estavam de acordo com os regulamentos estabelecidos pelos responsáveis da Comissão de Investigação Clínica e Ética e de acordo com a Declaração de Helsínquia da Associação Médica Mundial.

\section{CONFIDENCIALIDADE DOS DADOS}

Os autores declaram ter seguido os protocolos do seu centro de trabalho acerca da publicação de dados.

\section{CONSENTIMENTO INFORMADO}

Foi devidamente obtido o consentimento informado do doente.

\section{CONFLITOS DE INTERESSE}

Os autores declaram não ter qualquer conflito de interesse relativamente ao presente artigo.

\section{FONTES DE FINANCIAMENTO}

Não foi utilizada nenhuma bolsa ou subsídio para a realização do trabalho.

evaluation of suspected pulmonary embolism in the emergency department. Am J Emerg Med. 2008;26:181-5.

9. Hennelly KE, Baskin MN, Monuteuax MC, Hudgins J, Kua E, Commeree $A$, et al. Detection of pulmonary embolism in high-risk children. J Pediatr. 2016;178:214-8.

10. Biss TT, Brandão LR, Kahr WH, Chan AK, Williams S. Clinical features and outcome of pulmonary embolism in children. $\mathrm{Br} \mathrm{J} \mathrm{Haematol.}$ 2008;142:808-18

11. Agha BS, Sturm JJ, Simon HK, Hirsh DA. Pulmonary embolism in the pediatric emergency department. Pediatrics. 2013;132:663-7.

12. Hennelly KE, Baskin MN, Monuteuax MC, Hudgins J, Kua E, Commeree $A$, et al. Detection of pulmonary embolism in high-risk children. J Pediatr. 2016;178:214-8.

13. Kritsaneepaiboon S, Lee EY, Zurakowski D, Strauss KJ, Boiselle PM. MDCT pulmonary angiography evaluation of pulmonary embolism in children. Am J Roentgenol. 2009;192:1246-52.

14. Hancock HS, Wang M, Gist KM, Gibson E, Miyamoto SD, Mourani PM, et al. Cardiac findings and long-term thromboembolic outcomes following pulmonary embolism in children: a combined retrospectiveprospective inception cohort study. Cardiol Young. 2013;23:344-52.

15. Stam-Slob MC, Lambalk CB, van de Ree MA. Contraceptive and hormonal treatment options for women with history of venous thromboembolism. BMJ. 2015;351:h4847. 